\title{
A RELATION BETWEEN JACOBI AND LAGUERRE POLYNOMIALS
}

\section{EARL D. RAINVILLE}

1. Introduction. We shall obtain an apparently new expression for the Jacobi polynomial $P_{n}^{(\alpha, \beta)}(x)$ as a series of products of Laguerre polynomials of orders $\alpha$ and $\beta$ and of different arguments.

2. A relation between the Legendre and Laguerre polynomials. Because of the popularity of the Legendre and the simple Laguerre polynomials, we shall prove that special case of the relation between Jacobi and Laguerre polynomials. The method of proof carries through with no alteration in the general case and need not be repeated.

The generating function

$$
e^{t} J_{0}\left(2(t y)^{1 / 2}\right)=\sum_{n=0}^{\infty} L_{n}(y) \frac{t^{n}}{n !}
$$

for the Laguerre polynomials may be found in the standard books. For the Legendre polynomials a special case of a well known result ${ }^{1}$ of Bateman is

$$
I_{0}\left((2 t(x-1))^{1 / 2}\right) I_{0}\left((2 t(x+1))^{1 / 2}\right)=\sum_{n=0}^{\infty} P_{n}(x) \frac{t^{n}}{(n !)^{2}} .
$$

From (1) with $y=(1-x) / 2$ it follows, since $J_{0}(i y)=I_{0}(y)$, that

$$
e^{t} I_{0}\left((2 t(x-1))^{1 / 2}\right)=\sum_{n=0}^{\infty} L_{n}\left(\frac{1-x}{2}\right) \frac{t^{n}}{n !} .
$$

Using (1) with $y=(1+x) / 2$ and with $t$ replaced by $(-t)$, we may conclude that

$$
e^{-t} I_{0}\left((2 t(x+1))^{1 / 2}\right)=\sum_{n=0}^{\infty}(-1)_{0}{ }^{n} L_{n}\left(\frac{1+x}{2}\right) \frac{t^{n}}{n !} .
$$

Then, with the aid of the Cauchy product of two series, it may be seen that

Received by the editors September 19, 1944.

$1 \mathrm{H}$. Bateman, $A$ generalization of the Legendre polynomial, Proc. London Math. Soc. (2) vol. 3 (1905) pp. 111-123. See his formula (8). 


$$
\begin{aligned}
& I_{0}\left((2 t(x-1))^{1 / 2}\right) I_{0}\left((2 t(x+1))^{1 / 2}\right) \\
& =\sum_{n=0}^{\infty} \sum_{k=0}^{n}(-1)^{k} C_{n, k} L_{k}\left(\frac{1+x}{2}\right) L_{n-k}\left(\frac{1-x}{2}\right) \frac{t^{n}}{n !}
\end{aligned}
$$

where $C_{n, k}$ is the binomial coefficient.

Therefore, in view of (2), we have the desired result,

$$
P_{n}(x)=n ! \sum_{k=0}^{n}(-1)^{k} C_{n, k} L_{k}\left(\frac{1+x}{2}\right) L_{n-k}\left(\frac{1-x}{2}\right) .
$$

3. A similar formula for the Jacobi polynomial. From Bateman ${ }^{2}$ again we may obtain the following generating function for the Jacobi polynomial:

$$
\begin{aligned}
{\left[2^{\alpha+\beta} t^{-\alpha-\beta}(x-1)^{-\alpha}(x+1)^{-\beta}\right]^{1 / 2} I_{\alpha}\left((2 t(x-1))^{1 / 2}\right) I_{\beta}\left((2 t(x+1))^{1 / 2}\right) } \\
=\sum_{n=0}^{\infty} P_{n}^{(\alpha, \beta)}(x) \frac{t^{n}}{\Gamma(n+\alpha+1) \Gamma(n+\beta+1)} .
\end{aligned}
$$

For the Laguerre polynomial of order $\alpha$ it is known that

$$
e^{t}(y t)^{-\alpha / 2} J_{\alpha}\left(2(y t)^{1 / 2}\right)=\sum_{n=0}^{\infty} L_{n}^{(\alpha)}(y) \frac{t^{n}}{\Gamma(n+\alpha+1)} .
$$

These two results may be used just as we used (1) and (2) and they lead us to

$$
\begin{aligned}
& P_{n}^{(\alpha, \beta)}(x)=\sum_{k=0}^{n}(-1) \frac{\Gamma(n+\beta+1) \Gamma(n+\alpha+1)}{\Gamma(k+\beta+1) \Gamma(n-k+\alpha+1)} \\
& \cdot L_{k}^{(\beta)}\left(\frac{1+x}{2}\right) L_{n-k}^{(\alpha)}\left(\frac{1-x}{2}\right) .
\end{aligned}
$$

The UnIVersity of Michigan

2 Bateman, loc. cit. 